\title{
The HARPS search for southern extra-solar planets
}

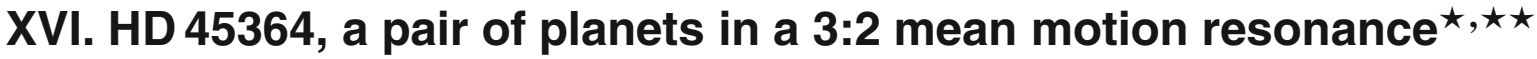

\author{
A. C. M. Correia ${ }^{1}$, S. Udry ${ }^{2}$, M. Mayor ${ }^{2}$, W. Benz ${ }^{3}$, J.-L. Bertaux ${ }^{4}$, F. Bouchy ${ }^{5}$, J. Laskar ${ }^{6}$, C. Lovis ${ }^{2}$, \\ C. Mordasini ${ }^{3}$, F. Pepe ${ }^{2}$, and D. Queloz ${ }^{2}$
}

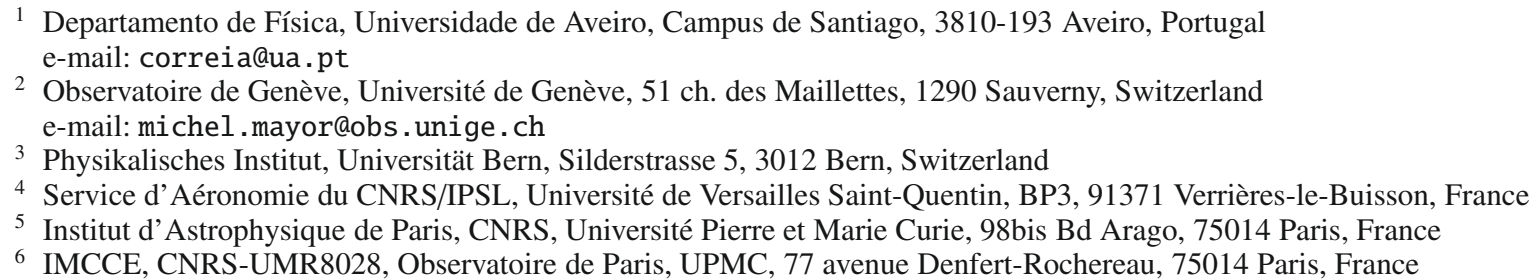

Received 8 August 2008 / Accepted 6 November 2008

\begin{abstract}
Precise radial-velocity measurements with the HARPS spectrograph reveal the presence of two planets orbiting the solar-type star HD 45364. The companion masses are $M \sin i=0.187 M_{\text {Jup }}$ and $0.658 M_{\text {Jup }}$, with semi-major axes of $a=0.681 \mathrm{AU}$ and $0.897 \mathrm{AU}$, and eccentricities of $e=0.168$ and 0.097, respectively. A dynamical analysis of the system further shows a 3:2 mean motion resonance between the two planets, which prevents close encounters and ensures the stability of the system over 5 Gyr. This is the first time that such a resonant configuration has been observed for extra-solar planets, although there is an analogue in our Solar System formed by Neptune and Pluto. This singular planetary system may provide important constraints on planetary formation and migration scenarios.
\end{abstract}

Key words. stars: individual: HD 45364 - stars: planetary systems - techniques: radial velocities - methods: observational

\section{Introduction}

At present, about $25 \%$ of the known exoplanets are in multiplanetary systems. The older the radial-velocity planet-search surveys, the higher the fraction of multi-planet families detected in these programs. Taking into account the still strong bias against long-period and/or low-mass planet detection, and the enhanced difficulty of fully characterizing systems with more than one planet, the fraction of known multi-planet systems is certainly still a lower limit. It appears likely that a high number, if not the majority, of planet-host stars form systems of planets rather than isolated, single planetary companions.

Among the known multi-planet systems, a significant fraction are in mean motion resonances, the majority of which are in low-order resonances. The 2:1 resonance is the most common (HD 73526, HD 82943, HD 128311, GJ 876), but other configurations are observed as well, such as the 3:1 resonance in HD 75732 or the 5:1 resonance in HD 202206. These resonances most probably arise from evolutionary processes as migrating planets forming in the protoplanetary disc become trapped. In our Solar System, we also find mean motion resonances in the

\footnotetext{
* Based on observations made with the HARPS instrument on the ESO $3.6 \mathrm{~m}$ telescope at La Silla Observatory under the GTO programme ID 072.C-0488.

$\star \star$ The table with the radial velocities is only available in electronic form at the CDS via anonymous ftp to

cdsarc.u-strasbg.fr $(130.79 .128 .5)$ or via

http://cdsweb.u-strasbg.fr/cgi-bin/qcat?]/A+A/496/521
}

satellites of the giant planets, or between these planets and several asteroids. The most well-known example is the Io-EuropaGanymede system in a 4:2:1 resonance, or the Neptune-Pluto system in a 3:2 resonance. While the satellites are believed to achieve resonant configurations after tidal evolution of their orbits, the resonances between planets and asteroids were probably formed after the inward or outward migration of the planets during the early stages of the evolution of the Solar System.

The presence of two or more interacting planets in a system dramatically increases our potential ability to constrain and understand the processes of planetary formation and evolution. The dynamical analysis of such systems is then very useful, first for constraining the system evolution history and second for determining the system "structure" in terms of orbital content.

Multi-planet systems are naturally found in planet-search programs. However, improving the precision of the radialvelocity measurements greatly helps their detection and complete characterization. The HARPS search for southern extrasolar planet is an extensive radial-velocity survey of some 2000 stars of the solar neighborhood conducted with the HARPS spectrograph on the ESO 3.6-m telescope at La Silla (Chile) in the framework of the Guaranteed Time Observations granted to the HARPS building consortium (Mayor et al. 2003). About half of the HARPS GTO time is dedicated to very high-precision measurements of non-active stars selected from the CORALIE planet-search program (Udry et al. 2000) and stars with already known giant planets, while searching for lower mass companions. This program reveals itself as very efficient in finding 
Table 1. Observed and inferred stellar parameters of HD 45364.

\begin{tabular}{llc}
\hline \hline Parameter & & HD 45364 \\
\hline Sp & & K0 V \\
$V$ & {$[\mathrm{mag}]$} & 8.08 \\
$B-V$ & {$[\mathrm{mag}]$} & 0.72 \\
$\pi$ & {$[\mathrm{mas}]$} & $30.69 \pm 0.81$ \\
$M_{\mathrm{V}}$ & {$[\mathrm{mag}]$} & 5.51 \\
$T_{\text {eff }}$ & {$[\mathrm{K}]$} & $5434 \pm 20$ \\
$\log g$ & {$[\mathrm{cgs}]$} & $4.38 \pm 0.03$ \\
{$[\mathrm{Fe} / \mathrm{H}]$} & {$[\mathrm{dex}]$} & $-0.17 \pm 0.01$ \\
$L$ & {$\left[L_{\odot}\right]$} & 0.57 \\
$M_{*}$ & {$\left[M_{\odot}\right]$} & $0.82 \pm 0.05$ \\
$v \sin i$ & {$\left[\mathrm{~km} \mathrm{~s}^{-1}\right]$} & 1 \\
$\log R_{\mathrm{HK}}^{\prime}$ & & -4.94 \\
$P_{\mathrm{rot}}\left(\log R_{\mathrm{HK}}^{\prime}\right)$ & {$[\mathrm{day}]$} & 32 \\
\hline
\end{tabular}

Photometric and astrometric data are from the Hipparcos catalogue (ESA 1997) and the stellar physical quantities from Sousa et al. (2008).

multi-Neptune (Lovis et al. 2006) and multi-Super Earth systems (Mayor et al. 2009).

From the HARPS high-precision survey, we present an interesting system of two planets in a 3:2 mean motion resonance around HD 45364, a configuration not observed previously among extra-solar planet, but similar to the Neptune-Pluto pair in the Solar System. Section 2 gathers useful stellar information about HD 45364, its derived orbital solution is described in Sect. 3, and the dynamical analysis of the system is discussed in Sect. 4. Finally, conclusions are drawn in Sect. 5.

\section{Stellar characteristics of HD 45364}

The basic photometric $(\mathrm{K} 0 \mathrm{~V}, V=8.08, B-V=0.72)$ and astrometric ( $\pi=30.69$ mas) properties of HD 45364 were taken from the Hipparcos catalogue (ESA 1997). They are recalled in Table 1 with inferred quantities such as the absolute magnitude $\left(M_{\mathrm{V}}=5.51\right)$ and the stellar physical characteristics derived from the HARPS spectra by Sousa et al. (2008). For the complete high-precision HARPS sample (including HD 45364), these authors provided homogeneous estimates of effective temperature $\left(T_{\text {eff }}=5434 \pm 20 \mathrm{~K}\right)$, metallicity $([\mathrm{Fe} / \mathrm{H}]=-0.17 \pm 0.01)$, and surface gravity $(\log g=4.38 \pm 0.03)$ of the stars. A projected low rotational velocity of the star, $v \sin i=1 \mathrm{~km} \mathrm{~s}^{-1}$ was derived from a calibration of the width of the cross-correlation function used in the radial-velocity estimate (Santos et al. 2002).

HD 45364 is a non-active star in our sample with an activity indicator $\log R_{H K}^{\prime}$ of -4.94 . No significant radial-velocity jitter is thus expected for the star. From the activity indicator, we also derive a stellar rotation period $P_{\text {rot }}=32$ day (following Noyes et al. 1984).

HD 45364 has a subsolar metallicity with $[\mathrm{Fe} / \mathrm{H}]=-0.17$ unlike most of the gaseous giant-planet host stars (Santos et al. 2004). According to simulations of planet formation based on the core-accretion paradigm, moderate metal-deficiency does not, however, prevent planet formation (Ida \& Lin 2004; Mordasini et al. 2008). Taking into account its subsolar metallicity, Sousa et al. (2008) derived a mass of $0.82 M_{\odot}$ for the star. From the color index, the derived effective temperature, and the corresponding bolometric correction, we estimated the star luminosity to be $0.57 L_{\odot}$.

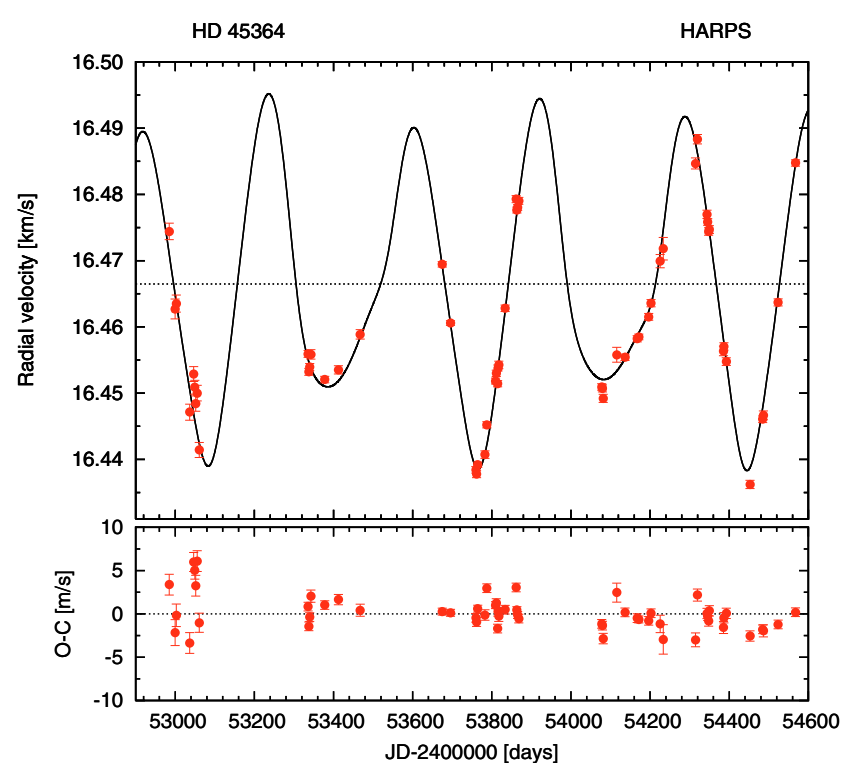

Fig. 1. HARPS radial velocities for HD 45364, superimposed on a 3-body Newtonian orbital solution (Table 2).

\section{Orbital solution for the HD 45364 system}

The HARPS observations of HD 45364 started in December 2003 and have now been going for about four years and a half. From the first stages of the observations, peculiar variations in the radial velocities (Fig. 1) have shown the presence of one or more companions in the system. After 58 measurements, we are now able to determine the nature of these bodies. Using a genetic algorithm combined with the iterative LevenbergMarquardt method (Press et al. 1992), we first attempted to fit the complete set of radial velocities using a model with two Keplerian orbits. This fit yields an adjustment of $\sqrt{\chi^{2}}=2.71$ and $\mathrm{rms}=1.38 \mathrm{~ms}^{-1}$ with two planets, one at $P=225.8$ day, $e=0.174$, and a minimum mass of $0.186 M_{\mathrm{Jup}}$, and another at $P=343.9$ day, $e=0.097$, and a minimum mass of $0.659 M_{\mathrm{Jup}}$.

Due to the proximity of the two planets and their high minimum masses, the gravitational interactions between these two bodies are strong. This prompts us to fit the observational data using a 3-body Newtonian model, assuming co-planar motion perpendicular to the plane of the sky, similarly to what has been done for the system HD 202206 (Correia et al. 2005). The orbital parameters corresponding to the the best fitted solution are listed in Table 2. We get identical results for $\sqrt{\chi^{2}}$ and velocity residuals as obtained with the two-Keplerian fit. Although there is no significant improvement in the fit, an important difference exists: the new orbital parameters for both planets show some deviations from the two-Keplerian case. In particular, the arguments of the periastrons $(\omega)$ show a difference of some degrees. We then conclude that, despite being unable to detect the planet-planet interaction in the present data, the orbits undergo important perturbations, and we expect to detect this gravitational interaction in the future. In Fig. 2, we plot the two best fit models evolving in time and clearly observe detectable deviations between the two curves that appear within ten years. The 3-body Newtonian fit provides a more accurate approximation of the HD 45364 planetary system, and the orbital parameters thus determined will be adopted as reference henceforward (Table 2).

We also fitted the data with a 3-body Newtonian model for which the inclination of the orbital planes, as well as the node of the outer planet orbit, were free to vary. We were able to find 
Table 2. Orbital parameters for the two bodies orbiting HD 45364, obtained with a 3-body Newtonian fit to observational data (Fig. 1).

\begin{tabular}{llcc}
\hline \hline Param. & [unit $]$ & HD 45364 b & HD 45364 c \\
\hline Date & {$[\mathrm{JD}-2400000]$} & \multicolumn{2}{c}{53500.00 (fixed) } \\
$V$ & {$\left[\mathrm{~km} \mathrm{~s}^{-1}\right]$} & $16.4665 \pm 0.0002$ \\
$P$ & {$[\mathrm{day}]$} & $226.93 \pm 0.37$ & $342.85 \pm 0.28$ \\
$\lambda$ & {$[\mathrm{deg}]$} & $105.76 \pm 1.41$ & $269.52 \pm 0.58$ \\
$e$ & & $0.1684 \pm 0.0190$ & $0.0974 \pm 0.012$ \\
$\omega$ & {$[\mathrm{deg}]$} & $162.58 \pm 6.34$ & $7.41 \pm 4.30$ \\
$K$ & {$[\mathrm{~m} / \mathrm{s}]$} & $7.22 \pm 0.14$ & $21.92 \pm 0.43$ \\
$i$ & {$[\mathrm{deg}]$} & 90 (fixed) & 90 (fixed) \\
\hline$a_{1} \sin i$ & {$\left[10^{-3} \mathrm{AU}\right]$} & 0.1485 & 0.6874 \\
$f(M)$ & {$\left[10^{-9} M_{\odot}\right]$} & 0.0085 & 0.3687 \\
$M \sin i$ & {$\left[M_{\text {Jup }}\right]$} & 0.1872 & 0.6579 \\
$a$ & {$[\mathrm{AU}]$} & 0.6813 & 0.8972 \\
\hline$N_{\text {meas }}$ & & \multicolumn{3}{c}{58} \\
Span & {$[\mathrm{day}]$} & \multicolumn{3}{c}{1583} \\
rms & {$[\mathrm{m} / \mathrm{s}]$} & \multicolumn{3}{c}{2.717} \\
$\sqrt{\chi^{2}}$ & & \multicolumn{2}{c}{} \\
\hline
\end{tabular}

Errors are given by the standard deviation $\sigma$ and $\lambda$ is the mean longitude of the date $(\lambda=\omega+M)$. The orbits are assumed co-planar.

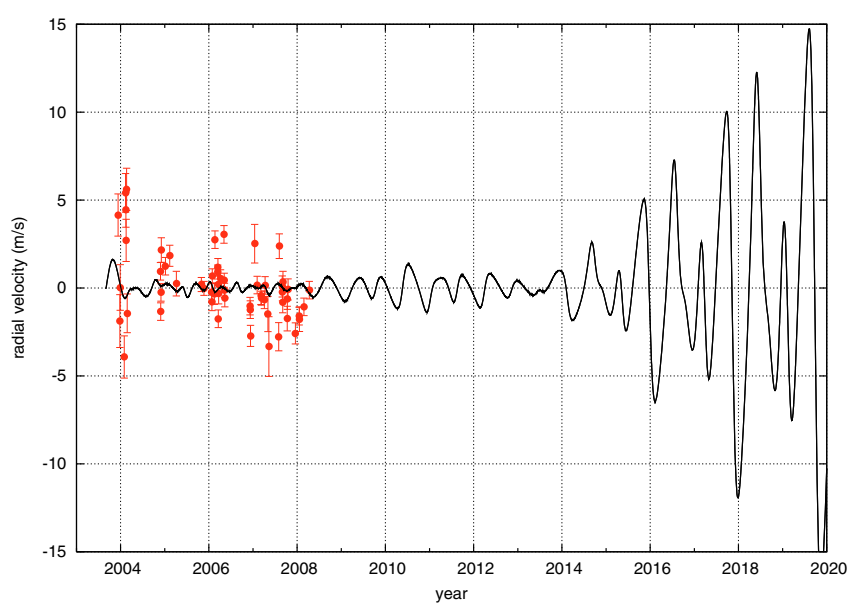

Fig. 2. Radial velocity differences between the two independent Keplerian model and the 3-body Newtonian model (Table 2). Data coincides at JD $=2453776$ (Feb. 9th 2006). We also plot the velocity residuals of the 3-body fit (Fig. 1). With the current HARPS's precision for this star, we expect to observe these differences within ten years.

a wide variety of configurations, some with low inclination values for one or both planets, that slightly improved our fit to a minimum $\sqrt{\chi^{2}}=2.32$ and $\mathrm{rms}=1.14 \mathrm{~m} / \mathrm{s}$. However, all of these determinations are uncertain, and since we also increase the number of free parameters by three, we cannot say that there has been an improvement with respect to the solution presented in Table 2. The inclination of the orbits therefore remain unknown, as do their true masses.

The residuals around the best fit solution are small, but remain slightly larger than the internal errors (Fig. 1). We may then ask if there are other companions with different orbital periods. For this purpose, we used a genetic algorithm, since we were unable to clearly isolate any significant peak in the frequency analysis of the residuals. The inclusion of additional companions in the system allows us to reduce the value of $\sqrt{\chi^{2}}$ slightly, although this can be justified as a natural consequence
Table 3. Fundamental frequencies for the orbital solution in Table 2.

\begin{tabular}{rrr}
\hline \hline & $\begin{array}{r}\text { Frequency } \\
o / y r\end{array}$ & $\begin{array}{r}\text { Period } \\
\mathrm{yr}\end{array}$ \\
\hline$n_{\mathrm{b}}$ & 576.429624 & 0.624534 \\
$n_{\mathrm{c}}$ & 384.033313 & 0.937419 \\
$g_{1}$ & -0.759309 & 474.115 \\
$g_{2}$ & 0.110472 & 3258.74 \\
$l_{\theta}$ & 19.820696 & 18.1628 \\
\hline
\end{tabular}

$n_{\mathrm{b}}$ and $n_{\mathrm{c}}$ are the mean motions, $g_{1}$ and $g_{2}$ are the secular frequencies of the periastrons, and $l_{\theta}$ is the libration frequency of the resonant angle $\theta_{\mathrm{b}}=2 \lambda_{\mathrm{b}}-3 \lambda_{\mathrm{c}}+\omega_{\mathrm{b}}$. Indeed, we have $2 n_{\mathrm{b}}-3 n_{\mathrm{c}}+g_{1}=0$.

of increasing the number of free parameters. Identical adjustments can be obtained with many orbital periods, as different as 5 or 18 days, frequently with very high eccentricity values. Therefore, no other companion can be conclusively detected in the residuals from the orbital solution listed in Table 2. The best fit solution was obtained by adding a linear drift to the data, with slope $=-0.86 \pm 0.09 \mathrm{~m} \mathrm{~s}^{-1} / \mathrm{yr}$, allowing us to reduce the value of $\sqrt{\chi^{2}}$ to 2.42 and the $\mathrm{rms}=1.21 \mathrm{~m} / \mathrm{s}$, while the orbital parameters of the two planets remain nearly the same. The solution in Table 2 must then be considered to be the best determination achievable so far, and a longer tracking of the system will provide more accurate orbital parameters for the HD 45364 system.

\section{Dynamical analysis}

We now briefly analyze the dynamics and stability of the planetary system given in Table 2. Due to the two planets' proximity and high values of the masses, we expect that both planets are affected by strong planetary perturbations from each other. The present orbits of the two planets almost cross (Fig. 6), and unless a resonant mechanism is present to avoid close encounters, the system cannot be stable.

\subsection{The 3:2 mean motion resonance}

The ratio between the orbital periods of the two planets determined by the fitting process (Table 2) is $P_{\mathrm{c}} / P_{\mathrm{b}}=1.511$, suggesting that the system may be trapped in a 3:2 mean motion resonance. To test the accurancy of this scenario, we performed a frequency analysis of the orbital solution listed in Table 2 computed over $100 \mathrm{kyr}$. The orbits of the planets are integrated with the symplectic integrator SABAC4 of Laskar \& Robutel (2001), using a step size of 0.02 years. We conclude that in the nominal solution of Table 2, the two planets in the HD 45364 system indeed show a 3:2 mean motion resonance, with resonant argument:

$\theta_{\mathrm{b}}=2 \lambda_{\mathrm{b}}-3 \lambda_{\mathrm{c}}+\omega_{\mathrm{b}}$

The fundamental frequencies of the systems are the two mean motions $n_{\mathrm{b}}$ and $n_{\mathrm{c}}$, the two secular frequencies of the periastrons $g_{1}$ and $g_{2}$, and the libration frequency of the resonant argument $l_{\theta}$ (Table 3 ). These frequencies are not independent because, due to the $3: 2$ resonance, we have up to the precision of the determination of the frequencies $\left(\approx 10^{-10}\right)$,

$2 n_{\mathrm{b}}-3 n_{\mathrm{c}}+g_{1}=0$.

The resonant argument $\theta_{\mathrm{b}}$ is in libration around $0^{\circ}$, with a libration period $2 \pi / l_{\theta}=18.16 \mathrm{yr}$, and an associated amplitude of about 68.44 degrees (Fig. 3a, Table 4). For the complete solution, the libration amplitude can reach more than 80 degrees 

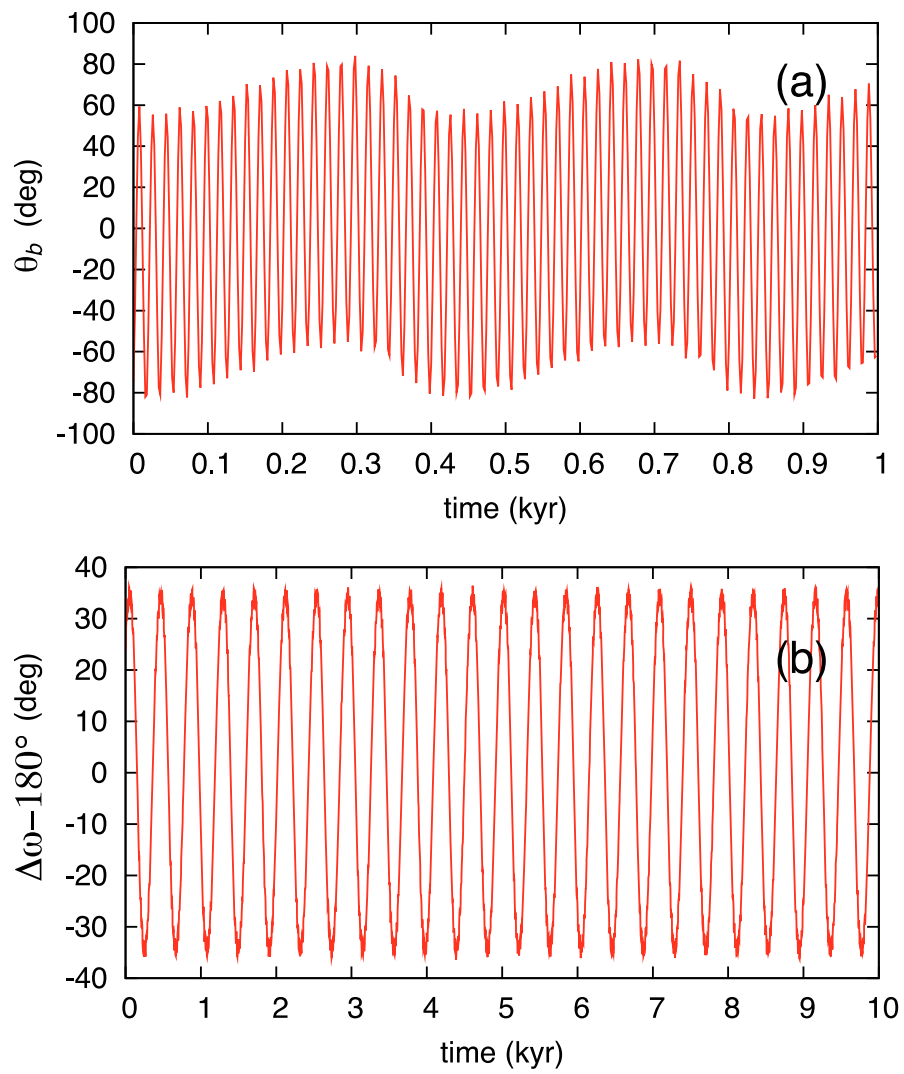

Fig. 3. Variation in the resonant argument, $\theta_{\mathrm{b}}=2 \lambda_{\mathrm{b}}-3 \lambda_{\mathrm{c}}+\omega_{\mathrm{b}}$ a) and in the secular argument, $\left.\Delta \omega=\omega_{\mathrm{b}}-\omega_{\mathrm{c}} \mathbf{b}\right)$, with time. $\theta_{\mathrm{b}}$ is in libration around $0^{\circ}$, with a libration period $P_{l_{\theta}} \simeq 18.16 \mathrm{yr}$, and a principal amplitude of about $68.4^{\circ}$ (Table 4). $\Delta \omega$ is in libration around $180^{\circ}$, with a libration frequency $g_{\Delta \omega}=g_{1}-g_{2}$ (corresponding to a period $P_{\Delta \omega} \simeq 413.9 \mathrm{yr}$ ), and a maximum amplitude of about $36.4^{\circ}$.

because additional periodic terms are present. In Table 4, we provide a quasi-periodic decomposition of the resonant angle $\theta_{\mathrm{b}}$ in terms of decreasing amplitude. All the quasi-periodic terms are easily identified as integer combinations of the fundamental frequencies (Table 3). Since the resonant angle is modulated by a relatively short period of about 18 years, the observation of the system over a few additional decades may provide an estimate of the libration amplitude and thus a strong constraint on the orbital parameters of the system.

Although the mean motions $n_{\mathrm{b}}$ and $n_{\mathrm{c}}$ can be associated with the two planets $b$ and $c$, respectively, it is not the case for the secular frequencies $g_{1}$ and $g_{2}$, and incidentally, both periastrons precess with mean frequency $g_{1}$ that is retrograde, with a period of 474.115 years. The two periastrons are thus locked in an antipodal state, and the difference $\Delta \omega=\omega_{\mathrm{b}}-\omega_{\mathrm{c}}$ is in libration around $180^{\circ}$ with an amplitude of about $36.4^{\circ}$ (Fig. 3b). As a result, the argument $\theta_{\mathrm{c}}=2 \lambda_{\mathrm{b}}-3 \lambda_{\mathrm{c}}+\omega_{\mathrm{c}}$ librates around $180^{\circ}$ with the same libration frequency $l_{\theta}$.

\subsection{Stability analysis}

To analyze the stability of the nominal solution and confirm the presence of the 3:2 resonance, we performed a global frequency analysis (Laskar 1993) in the vicinity of the nominal solution (Fig. 4), in the same way as achieved for the HD 202206 system by Correia et al. (2005). For each planet, the system is integrated on a regular 2D mesh of initial conditions, with varying
Table 4. Quasi-periodic decomposition of the resonant angle $\theta_{\mathrm{b}}=2 \lambda_{\mathrm{b}}-$ $3 \lambda_{\mathrm{c}}+\omega_{\mathrm{b}}$ for an integration over $100 \mathrm{kyr}$ of the orbital solution in Table 2.

\begin{tabular}{rrrrrrcr}
\hline \hline \multicolumn{7}{c}{ Combination } & \multicolumn{2}{c}{$v_{i}$} & $A_{i}$ & \multicolumn{1}{c}{$\phi_{i}$} \\
$n_{\mathrm{b}}$ & $n_{\mathrm{c}}$ & $g_{1}$ & $g_{2}$ & $l_{\theta}$ & \multicolumn{1}{c}{$(\mathrm{deg} / \mathrm{yr})$} & $(\mathrm{deg})$ & \multicolumn{1}{c}{$\mathrm{deg})$} \\
\hline 0 & 0 & 0 & 0 & 1 & 19.8207 & 68.444 & -144.426 \\
0 & 0 & -1 & 1 & 0 & 0.8698 & 13.400 & 136.931 \\
0 & 0 & 1 & -1 & 1 & 18.9509 & 8.606 & 168.643 \\
0 & 0 & -1 & 1 & 1 & 20.6905 & 8.094 & 82.505 \\
0 & 0 & -2 & 2 & 0 & 1.7396 & 2.165 & -176.138 \\
0 & 0 & -2 & 2 & 1 & 21.5603 & 0.622 & -50.564 \\
0 & 0 & 0 & 0 & 3 & 59.4621 & 0.540 & -73.279 \\
1 & -1 & 0 & 0 & -1 & 172.5756 & 0.506 & 7.504 \\
1 & -1 & 0 & 0 & 0 & 192.3963 & 0.501 & -46.923 \\
0 & 0 & -3 & 3 & 0 & 2.6093 & 0.416 & -129.207 \\
0 & 0 & 2 & -2 & 1 & 18.0811 & 0.420 & 121.712 \\
1 & -1 & 0 & 0 & 1 & 212.2170 & 0.416 & 78.651 \\
0 & 1 & -1 & 0 & 0 & 384.7926 & 0.451 & 176.155 \\
1 & -1 & 0 & 0 & -2 & 152.7549 & 0.424 & -118.070 \\
0 & 1 & -1 & 0 & -1 & 364.9719 & 0.341 & 50.581 \\
0 & 1 & -1 & 0 & 1 & 404.6133 & 0.274 & 121.729 \\
0 & 0 & 1 & -1 & 3 & 58.5923 & 0.212 & -120.210 \\
1 & -1 & 0 & 0 & 2 & 232.0377 & 0.201 & 24.225 \\
0 & 0 & -1 & 1 & 3 & 60.3319 & 0.211 & 153.652 \\
1 & 0 & -1 & 0 & -1 & 557.3682 & 0.182 & -86.342 \\
\hline
\end{tabular}

We have $\theta_{\mathrm{b}}=\sum_{i=1}^{N} A_{i} \cos \left(v_{i} t+\phi_{i}\right)$, where the amplitude and phases $A_{i}$, $\phi_{i}$ are given in degree, and the frequencies $v_{i}$ in degree/year. We only give the first 20 terms, ordered by decreasing amplitude. All terms are identified as integer combinations of the fundamental frequencies given in Table 3. The fact that we are able to express all the main frequencies of $\theta_{\mathrm{b}}$ in terms of exact combinations of the fundamental frequencies $g_{1}$, $g_{2}$ and $l_{\theta}$ is a signature of a very regular motion.

semi-major axis and eccentricity, while the other parameters are retained at their nominal values. The solution is integrated over $10 \mathrm{kyr}$ for each initial condition and a stability indicator is computed to be the variation in the measured mean motion over the two consecutive 5 kyr intervals of time. For regular motion, there is no significant variation in the mean motion along the trajectory, while it can vary significantly for chaotic trajectories. The result is reported in color in Fig. 4, where "red" represent the strongly chaotic trajectories, and "dark blue" the extremely stable ones. In both plots (Figs. 4a,b), it appears that the only stable zone that exists in the vicinity of the nominal solution are the stable 3:2 resonant zones.

It is quite remarkable that, in contrast to the findings of Correia et al. (2005) for the 5:1 resonance in the HD 202206 system, there is perfect coincidence between the stable 3:2 resonant islands, and curves of minimal $\chi^{2}$ obtained in comparing with the observations. Since these islands are the only stable zones in the vicinity, this picture presents a very coherent view of dynamical analysis and radial velocity measurments, which reinforces the confidence that the present system is in a 3:2 resonant state.

In Fig. 5, we plot the evolution of the HD 45364 planetary system over $100 \mathrm{kyr}$ in the rotating frame of the inner and the outer planet, respectively. Due to the 3:2 mean motion resonance trapping, the relative positions of the two planets are repeated and never become closer than about $0.37 \mathrm{AU}$, preventing close encounters and the consequent destruction of the system. The paths of the planets in the rotating frame illustrate the relationship between the resonance and the frequency of conjunctions with the internal or external planet. The inner planet is in a 3:2 resonance with the outer planet, so the orbital configuration of the system is repeated every 3 orbits of the inner planet and 

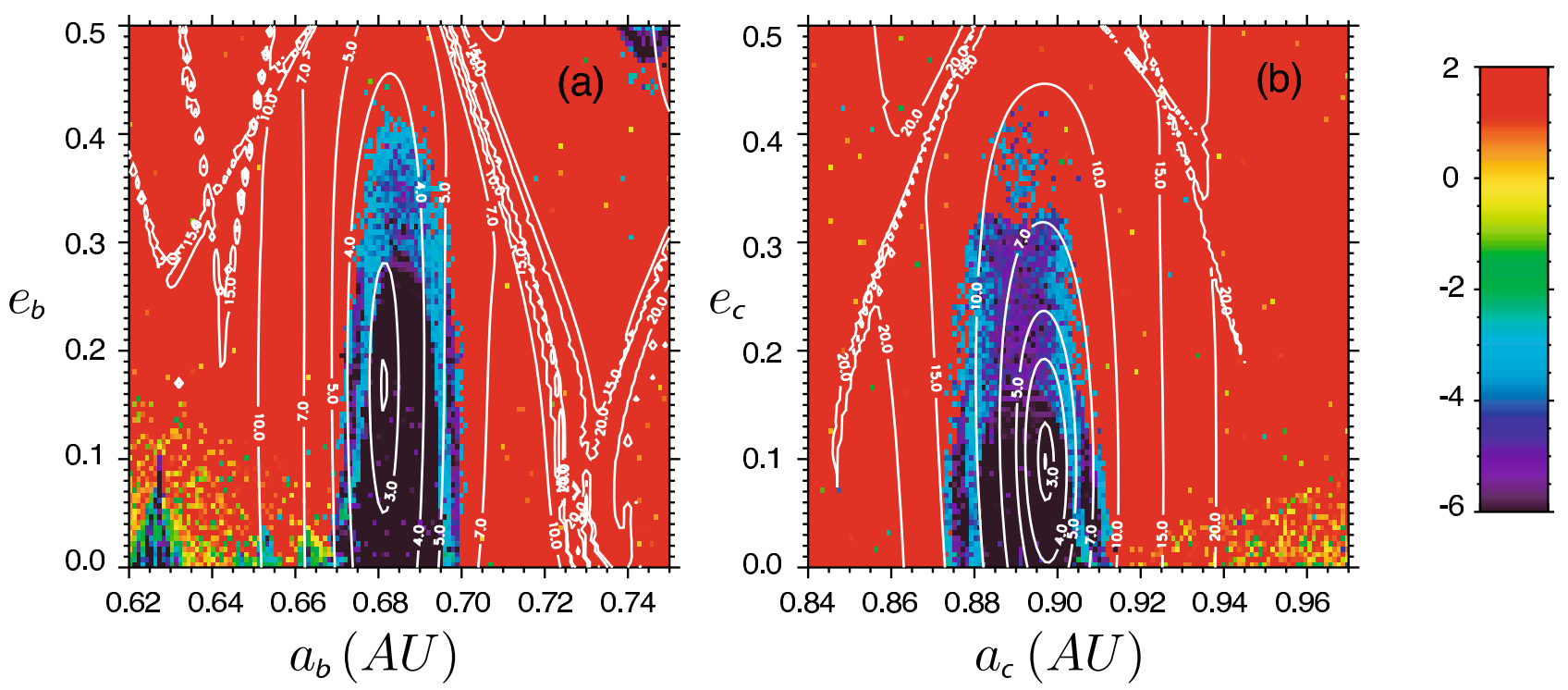

Fig. 4. Stability analysis of the nominal fit (Table 2) of the HD 45364 planetary system. For a fixed initial condition of the outer a) and inner planet b), the phase space of the system is explored by varying the semi-major axis $a_{\mathrm{k}}$ and eccentricity $e_{\mathrm{k}}$ of the other planet, respectively. The step size is $0.001 \mathrm{AU}$ in semi-major axis and 0.005 in eccentricity. For each initial condition, the full system is integrated numerically over 10 kyr and a stability criterion is derived with the frequency analysis of the mean longitude (Laskar 1990, 1993). As in Correia et al. (2005), the chaotic diffusion is measured by the variation in the frequencies. The "red" zone corresponds to highly unstable orbits, while the "dark blue" region can be assumed to be stable on a billion-years timescale. The contour curves indicate the value of $\chi^{2}$ obtained for each choice of parameters. It is remarkable that in the present fit, there is perfect correspondence between the zone of minimal $\chi^{2}$ and the 3:2 stable resonant zone, in "dark blue".
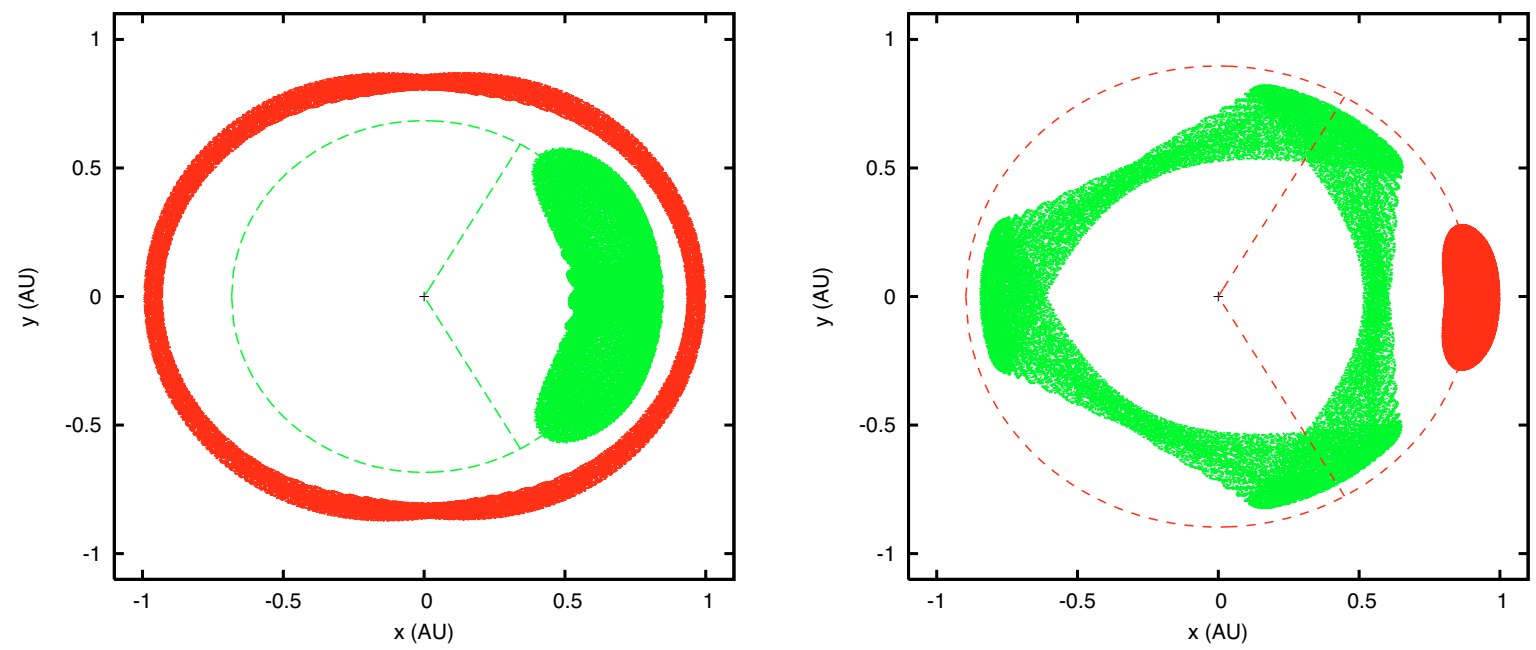

Fig. 5. Evolution of the HD 45364 planetary system over $100 \mathrm{kyr}$ in the co-rotating frame of the inner planet (left) and outer planet (right). $x$ and $y$ are spatial coordinates in a frame centered on the star and rotating with the frequency $n_{\mathrm{b}}(l e f t)$ or $n_{\mathrm{c}}$ (right). Due to the 3:2 mean motion resonance the planets never get too close, the minimal distance of approach being $0.371 \mathrm{AU}$, while the maximum distance can reach $1.811 \mathrm{AU}$. We also observe that the trajectories are repeated every 3 orbits of the inner planet and every 2 orbits of the outer planet.

every 2 orbits of the outer planet. In this particular frame, we are also able to see the libration of each planet around its equilibrium position.

\subsection{Orbital evolution}

From the previous stability analysis, it is clear that the HD 45364 planetary system listed in Table 2 is trapped in a 3:2 mean motion resonance and stable over a Gyr timescale. Nevertheless, we tested directly this by performing a numerical integration of the orbits over 5 Gyr using the symplectic integrator SABAC4 of Laskar \& Robutel (2001) with a step size of 0.02 years. The results displayed in Fig. 6 show that the orbits indeed evolve in a regular way, and remain stable throughout the simulation, which corresponds to the estimated age of the star.

Because of the strong gravitational interactions between the two planets, both orbital eccentricities present significant variations. The eccentricity of the inner planet is within $0.12<e_{\mathrm{b}}<$ 0.29 , while that of the outer planet is within $0.04<e_{\mathrm{c}}<0.13$. We also observe rapid secular variations in the orbital parameters, mostly driven by the rapid secular frequency $g_{1}$, of period $2 \pi / g_{1} \approx 474 \mathrm{yr}$ (Table 3 ). These secular variations in the orbital elements occur much more rapidly than in our Solar System, which should enable them to be detected directly from observations. 


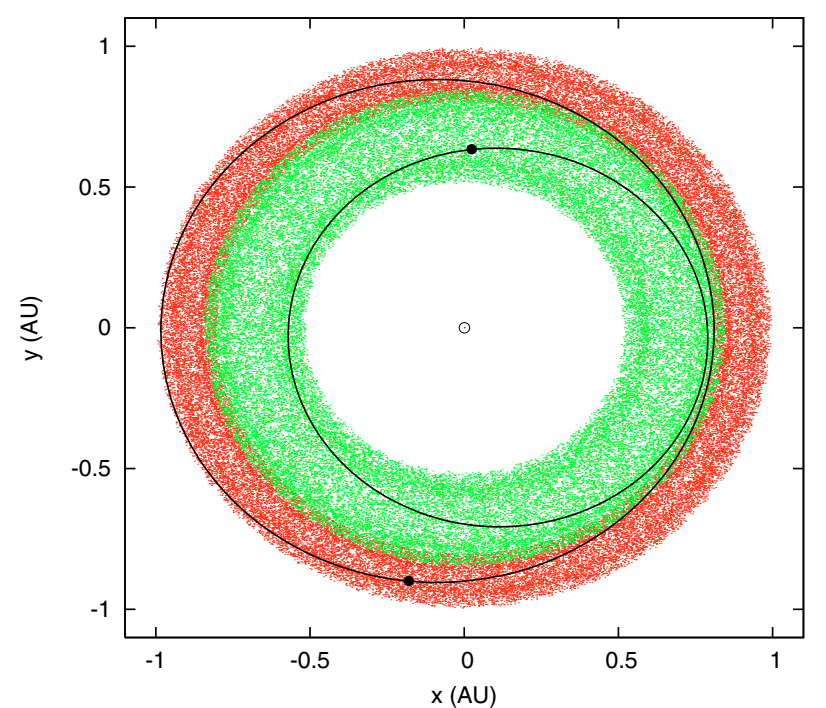

Fig. 6. Long-term evolution of the HD 45364 planetary system over 5 Gyr starting with the orbital solution from Table 2. The panel shows a face-on view of the system. $x$ and $y$ are spatial coordinates in a frame centered on the star. Present orbital solutions are traced with solid lines and each dot corresponds to the position of the planet every $100 \mathrm{kyr}$. The semi-major axes (in AU) are almost constant $\left(0.676<a_{\mathrm{b}}<0.693\right.$ and $\left.0.893<a_{\mathrm{c}}<0.902\right)$, but the eccentricities undergo significant variations $\left(0.123<e_{\mathrm{b}}<0.286\right.$ and $\left.0.042<e_{\mathrm{c}}<0.133\right)$. The fundamental periods related to the precession of the periastrons are $2 \pi / g_{1}=-474 \mathrm{yr}$ and $2 \pi / g_{2}=3259 \mathrm{yr}$.

\section{Discussion and conclusion}

We have reported the detection of two planets orbiting the star HD 45364, with orbital periods of 228 and 342 days, and minimum masses of 0.187 and $0.658 M_{\text {Jup }}$, respectively. A dynamical analysis of the system has further shown a 3:2 mean motion resonance between the two planets, which ensures its stability over 5 Gyr despite the proximity of the two orbits. This is the first time that such an orbital resonant configuration has been observed for extra-solar planets, although an analogue does exist in our Solar System composed by Neptune and Pluto. However, while Neptune evolves in an almost circular orbit and is much more massive than Pluto (which is the largest member of the asteroid family of the Plutinos), the two planets around HD 45364 have masses comparable to those of Saturn and Jupiter, and are evolving in orbits with moderate eccentricity.
Dynamically, the system is extremely interesting. In the nominal solution, the resonant angle $\theta_{\mathrm{b}}=2 \lambda_{\mathrm{b}}-3 \lambda_{\mathrm{c}}+\omega_{\mathrm{b}}$ is in libration around $0^{\circ}$, with a libration period of 18.16 years and a dominating amplitude of 68.44 degrees. Such an orbital configuration may have been reached through the dissipative process of planet migration during the early stages of the system evolution. However, after being captured in resonance, subsequent migration produces a significant increase in planetary eccentricities, unless a damping mechanism is applied. Since the eccentricities of the two planets around HD 45364 are relatively small (Table 2), migration may cease shortly after capture in resonance occurs, or, according to Crida et al. (2008), an inner disc must be present. This singular planetary system may then provide important constraints on planetary formation and migration scenarios.

The strong gravitational interactions between the planets may also allow us to model their effect more accurately in the near future. With the current precision of HARPS, about $1 \mathrm{~m} / \mathrm{s}$ for HD 45364, we expect to detect the signature of planet-planet interactions in data in a few decades. The planet-planet interactions may provide important information about the inclination of the orbital planes and allow us to determine the precise masses of both planets.

Acknowledgements. We acknowledge support from the Swiss National Research Found (FNRS), the Geneva University, Fundação Calouste Gulbenkian (Portugal), and French CNRS.

\section{References}

Correia, A. C. M., Udry, S., Mayor, M., et al. 2005, A\&A, 440, 751 Crida, A., Sándor, Z., \& Kley, W. 2008, A\&A, 483, 325

ESA. 1997, VizieR Online Data Catalog, 1239

Ida, S., \& Lin, D. N. C. 2004, ApJ, 616, 567

Laskar, J. 1990, Icarus, 88, 266

Laskar, J. 1993, Physica D Nonlinear Phenomena, 67, 257

Laskar, J., \& Robutel, P. 2001, Celestial Mechanics and Dynamical Astronomy, 80,39

Lovis, C., Mayor, M., Pepe, F., et al. 2006, Nature, 441, 305

Mayor, M., Pepe, F., Queloz, D., et al. 2003, The Messenger, 114, 20

Mayor, M., Udry, S., Lovis, C., et al. 2009, A\&A, 493, 639

Mordasini, C., Alibert, Y., Benz, W., \& Naef, D. 2008, ASP Conf., 398, 235

Noyes, R. W., Hartmann, L. W., Baliunas, S. L., Duncan, D. K., \& Vaughan, A. H. 1984, ApJ, 279, 763

Press, W. H., Teukolsky, S. A., Vetterling, W. T., \& Flannery, B. P. 1992, Numerical recipes in FORTRAN. The art of scientific computing (Cambridge: University Press, 2nd ed.)

Santos, N. C., Israelian, G., \& Mayor, M. 2004, A\&A, 415, 1153

Santos, N. C., Mayor, M., Naef, D., et al. 2002, A\&A, 392, 215

Sousa, S. G., Santos, N. C., Mayor, M., et al. 2008, A\&A, 487, 373

Udry, S., Mayor, M., Naef, D., et al. 2000, A\&A, 356, 590 